\title{
Predictive value of lexical-semantic processing in the early diagnosis of alzheimer's disease. a case report
}

\begin{abstract}
The cognitive decline associated with Alzheimer's disease (AD) compromises virtually all mental functions of the individual, however, the cognitive abilities that are most affected since the onset of the disease are memory and language. The following article presents a case of a 72-year-old man with incipient AD traces, who was evaluated using a cognitive performance protocol, a daily-activity scale, and a severity rating scale for dementia. The objective of the evaluation was to characterize performance in lexical-semantic abilities of the patient that allows to visualize clinically the predictive value of these linguistic functions. The results obtained from the evaluation allow to estimate the high discriminative value of linguistic domains such as verbal fluency and denomination in early stages of AD.
\end{abstract}

Keywords: alzheimer's disease, cognitive functions, lexical-semantic level
Volume I Issue 5 - 2017

\author{
Pamela San Martín Cofré,' Carlos Rojas \\ Zepeda, ${ }^{1,2}$ Karla Salazar Céspedes ${ }^{3}$ \\ 'Department of Health Rehabilitation Sciences, University of \\ Bio-Bio, Chile \\ ${ }^{2}$ Linguistics Doctoral Program, University of Concepción, Chile \\ ${ }^{3}$ English Teaching Training Program, Adventist University, Chile
}

Correspondence: Pamela San Martín Cofré, Department of Health Rehabilitation Sciences, University of Bio-Bio, Fernando May Campus, Chile, Tel (56-42)2463245,

Email msanmartin@ubiobio.cl

Received: August 09, 2017 | Published: August 28, 2017

\section{Introduction}

Alzheimer's disease is the most common form of dementia. Characterized by an insidious onset and progressive course, its occurrence takes place mainly in old age. This condition manifests itself through cognitive, behavioral and functional decay. ${ }^{1}$ This cognitive decline that occurs in AD compromises practically all mental functions, however, those that are most affected since the beginning of the disease are memory and language ${ }^{2}$ where the main alterations are in the Semantic fluency and denomination. ${ }^{3}$ However, there are also difficulties in semantic tasks of lexical decision and semantic coding, among others. ${ }^{4}$ Therefore, knowing the lexical-semantic performance in the aging stage could contribute to the early diagnosis of $\mathrm{AD}$ given the predictive value that these linguistic domains may present ${ }^{1,2,4}$ considering that timely detection and treatment of $\mathrm{AD}$ increases the probability of delaying its inevitable progression. ${ }^{1}$

\section{Case report}

This article presents the case of a 72-year-old male having 12 years of schooling, who comes to the Clinic of Human Communication Studies at University of Bio-Bio, by referral of Neurologist, with diagnosis of Alzheimer's disease in the initial stage.In order to measure the patient's cognitive performance, the Chilean version of the Test for Early Detection of Alzheimer's Disease (TDPEA-Ch) was administered and the Chilean version of the Addenbrooke-Revised Cognitive Examination was used as a referential frame (ACE-RCh) ). The Barthel Index was applied to assess the functionality of activities of daily living (ADL). Finally, the severity of dementia was categorized by the means of the Clinical Dementia Rating Scale (CDR).

The results of the clinical evaluation with respect to the CDR protocol allowed to classify the subject in a stage of mild dementia evolution, because it obtained 1 point, of a scale of 5 , in which CDR 0 denotes absence of cognitive alteration, CDR 0.5 expresses very mild dementia, CDR 1 indicates mild dementia, CDR 2 shows moderate dementia and CDR 3 indicates severe dementia. The Barthel Index showed 75 points out of a total of 100, placing it in a state of mild dependency in activities of daily life, needing supervision in some activities such as personal hygiene tasks, dressing and grooming. As for the performance in the 10 tasks of TDPEA-Ch, difficulties are observed, obtaining a score of 30 out of 100 , failing to obtain the maximum score of 10 (one point per item) in none of them. The tasks evaluated are:1) semantic verbal fluency, (name fruits), where he obtained 4 points; 2 ) phonological verbal fluency (words beginning with the letter " $\mathrm{f}$ ") with 5 points; 3 ) verbal fluency of proper names (public characters) with 2 points; 4) denomination to the definition, with 6 points; 5) denomination of objects by visual confrontation, with 5 points; 6) name of proper names, with 4 points; 7) immediate recall of a list of words, with 2 points; 8) deferred memory of a list of words, with 0 points; 9 ) immediate recall of data of a person, with 2 points; and 10) deferred memory of a person's data, with 0 points. The performance of the various TDPEA-Ch tasks are congruent with the low cognitive efficiency scores of the ACE-R-Ch test in language subtests (18/26 points), memory ( $7 / 26$ points), verbal fluency $(5 / 14$ points) and with the global cognitive efficiency score of this test (total score 53/100).

\section{Discussion}

Firstly, the low performance reported in lexical-semantic skills allows us to highlight and confirm the discriminative value of these linguistic domains in the early stages of $\mathrm{AD}$, which is in agreement with what has been reported in the literatura regarding the linguistic performance in the early stages of this dementia in lexical-semantic tasks such as verbal fluency ${ }^{5}$ denomination of objects by visual confrontation $^{6}$ denomination of famous characters ${ }^{7}$ and in some memory tasks, such as immediate recall ${ }^{5}$ and delayed recall. ${ }^{8}$

Second, data provided by tests such as ACE-R-CH and TDPEA$\mathrm{CH}$ as a whole allow us to recognize a profile for linguistic alterations in $\mathrm{AD}$, where the proper evaluation of the lexical-semantic domain of the individual can contribute and give clues to complement the early diagnosis of $\mathrm{AD}$; Although sociodemographic factors such as age, occupation and socio-cultural level, as well as psycholinguistic variables of words; Can influence and generate bias in the results obtained. 
Third, considering the population aging and the importance of having new instruments that complement the existing neuropsychological tests, it is observed the need to develop studies with population groups allowing to know the linguistic-communicative profile of this disease in the different stages of evolution, so as to offer therapeutic alternatives that allow to compensate the deficit of the language and to improve the quality of life of the users and their caregivers on the basis of valid clinical evidence and with proven results.

\section{Acknowledgements}

None.

\section{Conflict of interest}

The author declares no conflict of interest.

\section{References}

1. Valls PC, Molinuevo JL, Rami L. Diagnóstico precoz de la enfermedad de Alzheimer: fase prodrómica y preclínica. Neurol. 2010;51:471-480.

2. Cuetos VF, Menéndez GM, Calatayud NT. Descripción de un nuevo test para la detección precoz de la enfermedad de Alzheimer. REV NEUROL. 2007;44(8):469-474.
3. Facal D, González M, Buiza C, et al. Envejecimiento, deterioro cognitivo y lenguaje: Resultados del Estudio Longitudinal Donostia. Revista de Logopedia, Foniatría Y Audiología. 2009;29(1):4-12.

4. Hernández JJ. Demencias: los problemas de lenguaje como hallazgos tempranos. Acta Neurol Colomb. 2010;26:101-111.

5. Cuetos F, Martínez T, Martínez C, et al. Lexical processing in Spanish patients with probable Alzheimer's disease. Cogn Brain Res. 2003;17(3):549-561.

6. Cuetos F, Rodríguez FJ, Menéndez M. Semantic markers in the diagnosis of neurodegenerative dementias. Dement Geriatr Cogn Disord. 2009;28(3):267-274

7. Garrard P, Maloney L, Hodges J, et al. The effects of very early Alzheimer's disease on the characteristics of writing by a renowned author. Brain. 2005;128(2):250-260.

8. Chen P, Ratcliff G, Belle SH, et al. Ganguli M. Cognitive tests that best discriminate between presymptomatic $\mathrm{AD}$ and those who remain nondemented. Neurology. 2000;55(12):1847-1853. 